\title{
Discrete Signaling for Non-Coherent, Single-Antenna, Rayleigh Block-Fading Channels
}

Marcin Pikus ${ }^{12}$, Gerhard Krammer², and Georg Böcherer ${ }^{2}$

'Huawei Technologies Duesseldorf GmbH, Germany $\quad$ Institute for Communications Engineering, Technical University of Munich, Germany

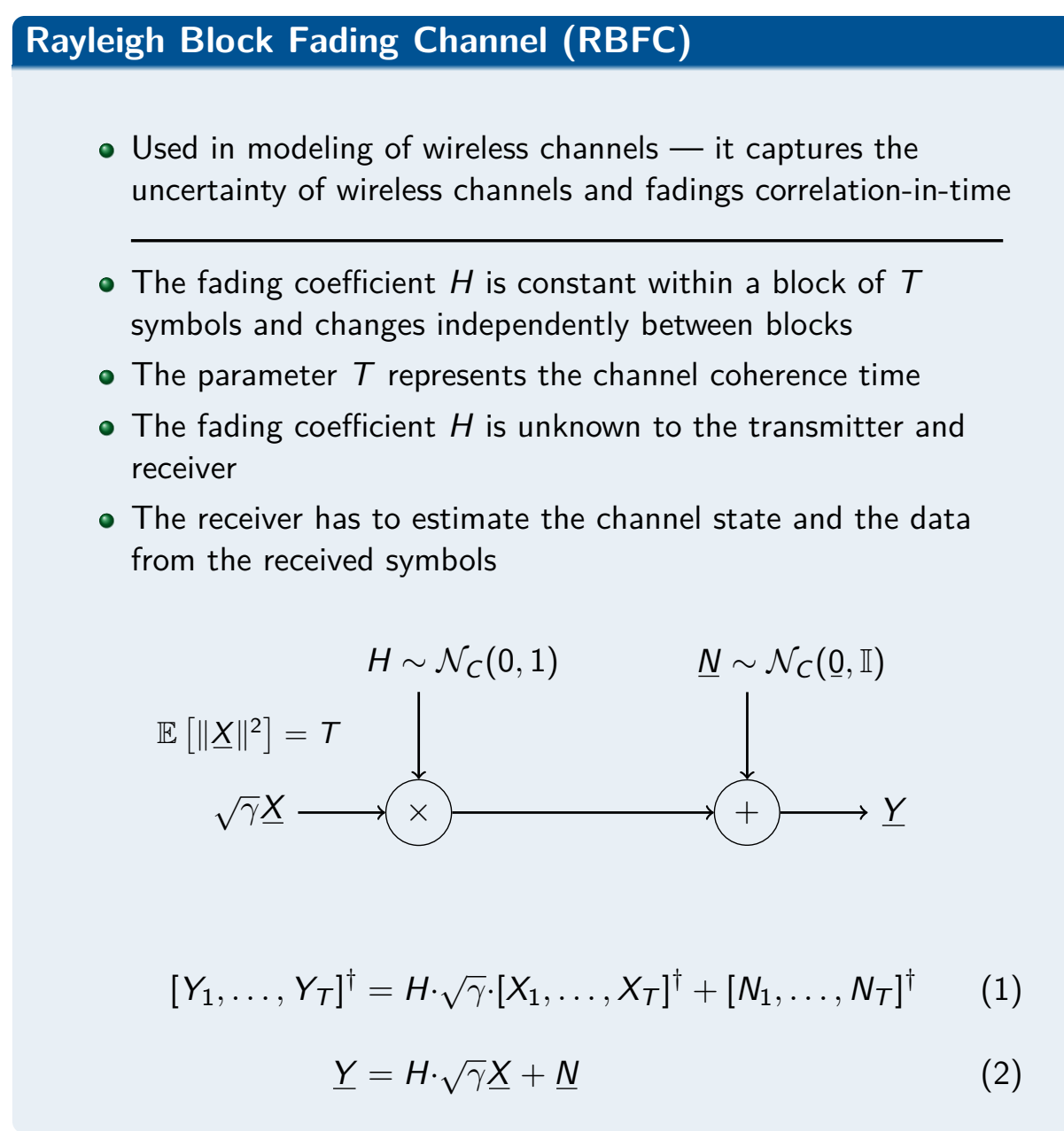

\section{Signaling in the Literature}

CSI Capacity When the receiver knows $H$, Gaussian IID input signal $X \sim \mathcal{N}_{C}(0, I)$ achieves the ca
upper-bounds all non-coherent rates

Gaussian IID When the receiver does not know $H$, Gaussian IID input signal $\underline{X} \sim \mathcal{N}_{C}(\underline{0}, \mathbb{I})$ in not capacity-achieving. However it performs well for large $T[1]$

Pilot schemes The transmitter inserts a fixed pilot symbol inside each fading block. At the receiver the pilot symbol is used to decode the data [2].

USTM Unitary Space-Time Modulation transmits in each fading $T$-dimensional complex sphere with radius $\sqrt{T}$

\section{Experimental Signaling}

Discrete Product Form is the proposed discrete signaling scheme (n)

On-Off USTM is an extension of the USTM. The scheme transmits either a vector $\Phi$ as in USTM or a vector of
0 's. We optimize the probability $P_{0}$ of transmitting the Q-symbol. This scheme is an isotropicaly (continuous) counterpart of DP

On-Off Gaussian is an extension of Gaussian IID signaling. The scheme transmits either a vector of Gaussian IID RV transmitting the 0 -symbol.

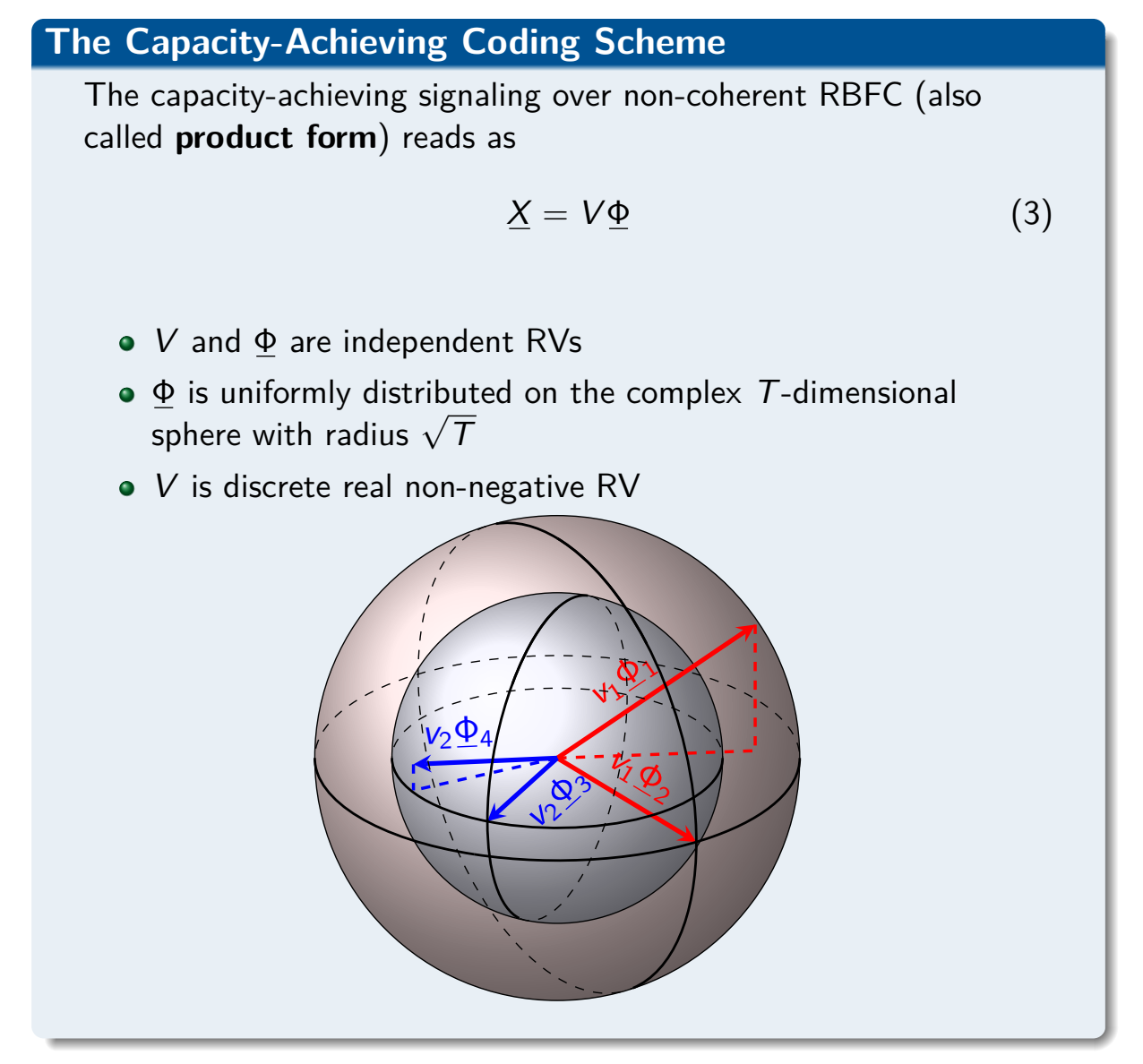

\section{Discretization}

(1) Choose spheres radius, i.e., choose RV $V$

$$
V= \begin{cases}0, & \text { with probability } 1-P_{1} \\ v_{1}=\sqrt{\frac{1}{P_{1}}}, & \text { with probability } P_{1}\end{cases}
$$

(2) Sample the spheres by choosing the following points

$$
\underline{\Theta}=\left[\Theta_{1}, \ldots, \Theta_{T}\right]
$$

where $\Theta_{i}$ are IID uniformly distributed RVs on the QPSK alphabet $\mathcal{A}_{4}=\{1,-1, j,-j\}$

$$
\begin{aligned}
\text { We get the Discrete Product Form scheme } \\
\qquad \underline{X}= \begin{cases}0, & \text { with probability } 1-P_{1} \\
\sqrt{\frac{1}{P_{1}}} \Theta & \text { with probability } P_{1},\end{cases}
\end{aligned}
$$

and $P_{1}$ is chosen to maximize the mutual information.

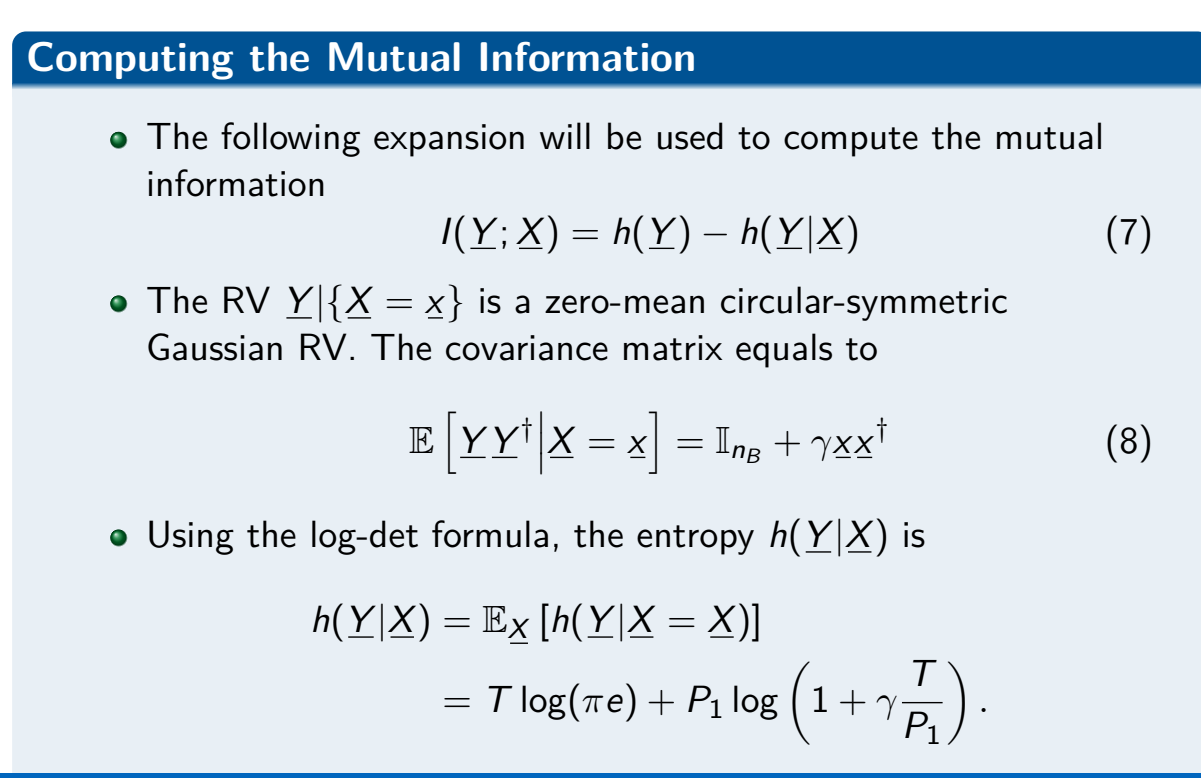

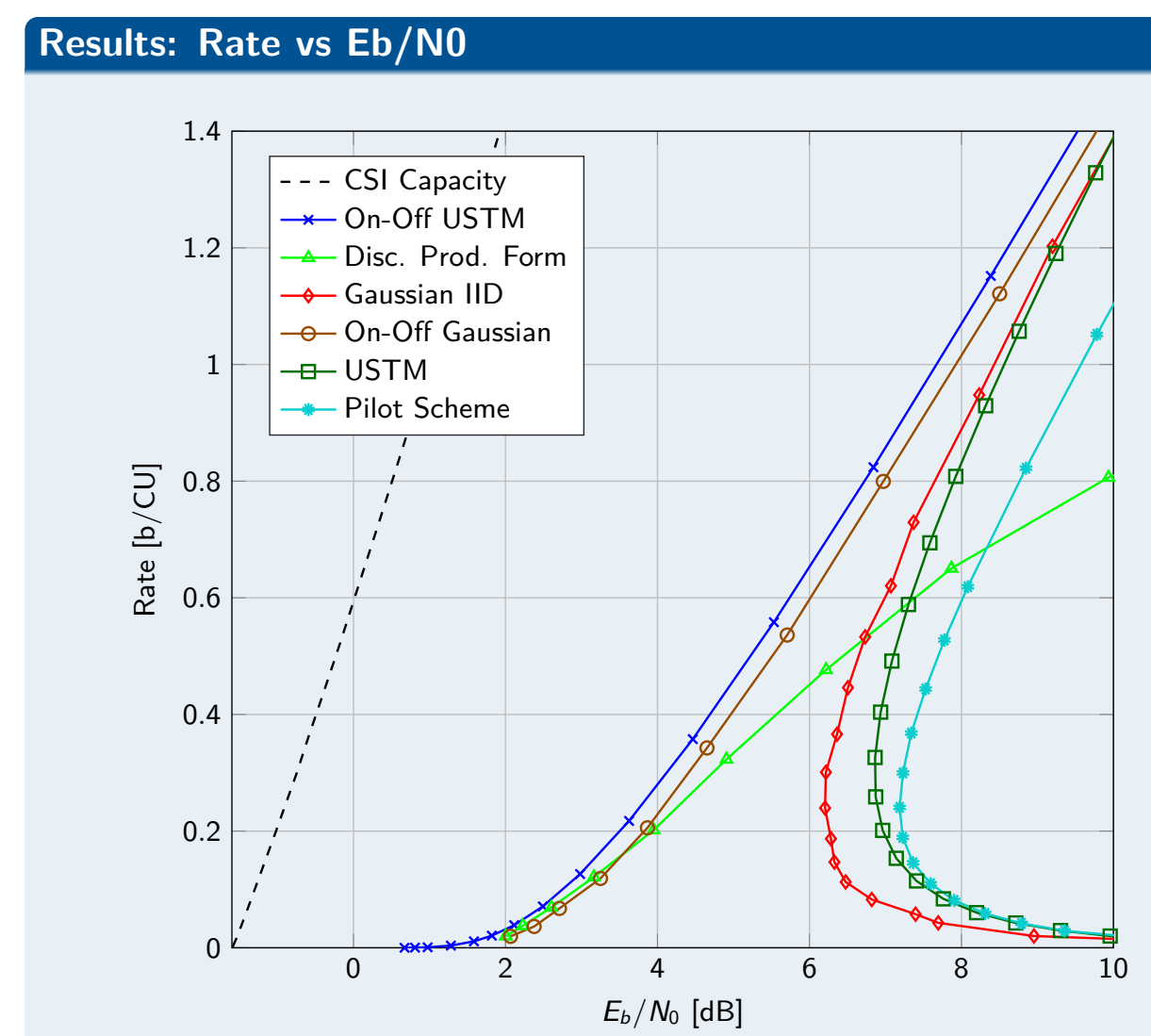

Information rates for block length $T=2$

\section{Computing $h(\underline{Y})$}

- The following formula can be used to compute $h(\underline{Y})$

$h(\underline{Y})=\mathbb{E}_{\underline{Y}}[-\log p(\underline{Y})]$. - The knowledge of $p(y)$ is needed to evaluate (9). However, a
direct computation fails due to exponential complexity growth in block length $T$

$$
p(\underline{y})=\sum_{\underline{x}} P(\underline{x}) p(\underline{y} \mid \underline{x})
$$

Instead, we use the fact that $\underline{Y}$ conditioned on $V$ and $H$ is a

$$
p(\underline{y})=\operatorname{Pr}(V=0) \underbrace{p(y \mid V=0)}_{\sim \mathcal{N}_{C}(0, \mathbb{I})}+\operatorname{Pr}\left(V=v_{1}\right) p\left(\underline{y} \mid V=v_{1}\right) \quad(11)
$$

Then the second term

$$
\begin{aligned}
p\left(\underline{y} \mid v_{1}\right) & =\mathbb{E}_{H}\left[p\left(\underline{y} \mid v_{1}, H\right)\right] \\
& =\mathbb{E}_{H}\left[\prod_{i=1}^{T} p\left(y_{i} \mid v_{1}, H\right)\right] \\
& =\mathbb{E}_{H}\left[\prod_{i=1}^{T} \sum_{\theta_{i} \in \mathcal{A}_{4}} P\left(\theta_{i}\right) p\left(y_{i} \mid \theta_{i}, v_{1}, H\right)\right] \\
& =\mathbb{E}_{H}\left[\prod_{i=1}^{T} \sum_{\theta_{i} \in \mathcal{A}_{4}} \frac{1}{4} \cdot \frac{1}{\pi} e^{-\left.\left|y_{i}-H \sqrt{\gamma} \theta_{i}\right|_{1}\right|^{2}}\right] \\
& =\mathbb{E}_{H}\left[f\left(\underline{y}, v_{1}, H\right)\right]
\end{aligned}
$$

The complexity of computing $f\left(\underline{y}, v_{1}, H\right)$ grows linearly wit respect to the block size $T$. To evaluate the expectation (12) we apply MC averaging.

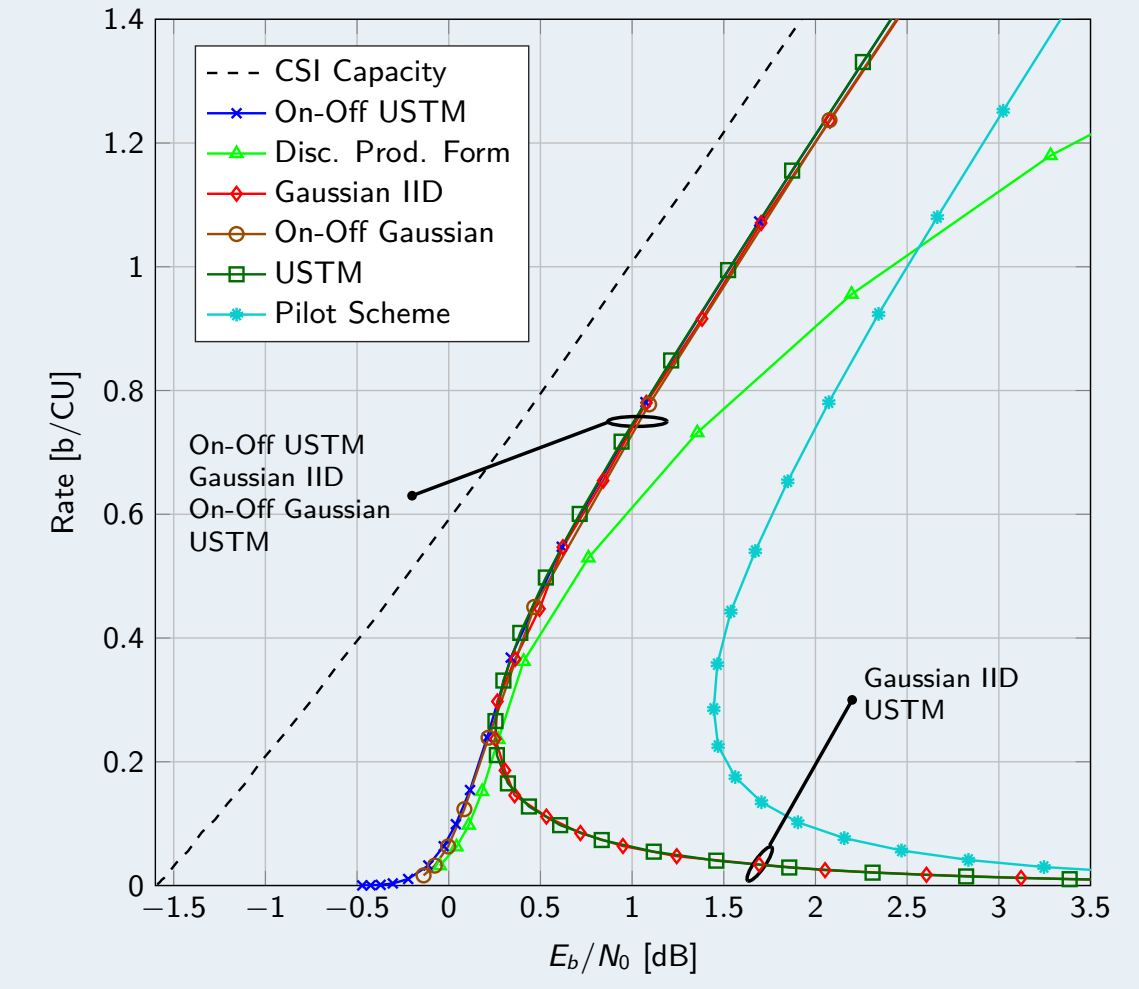

Information rates for block length $T=50$

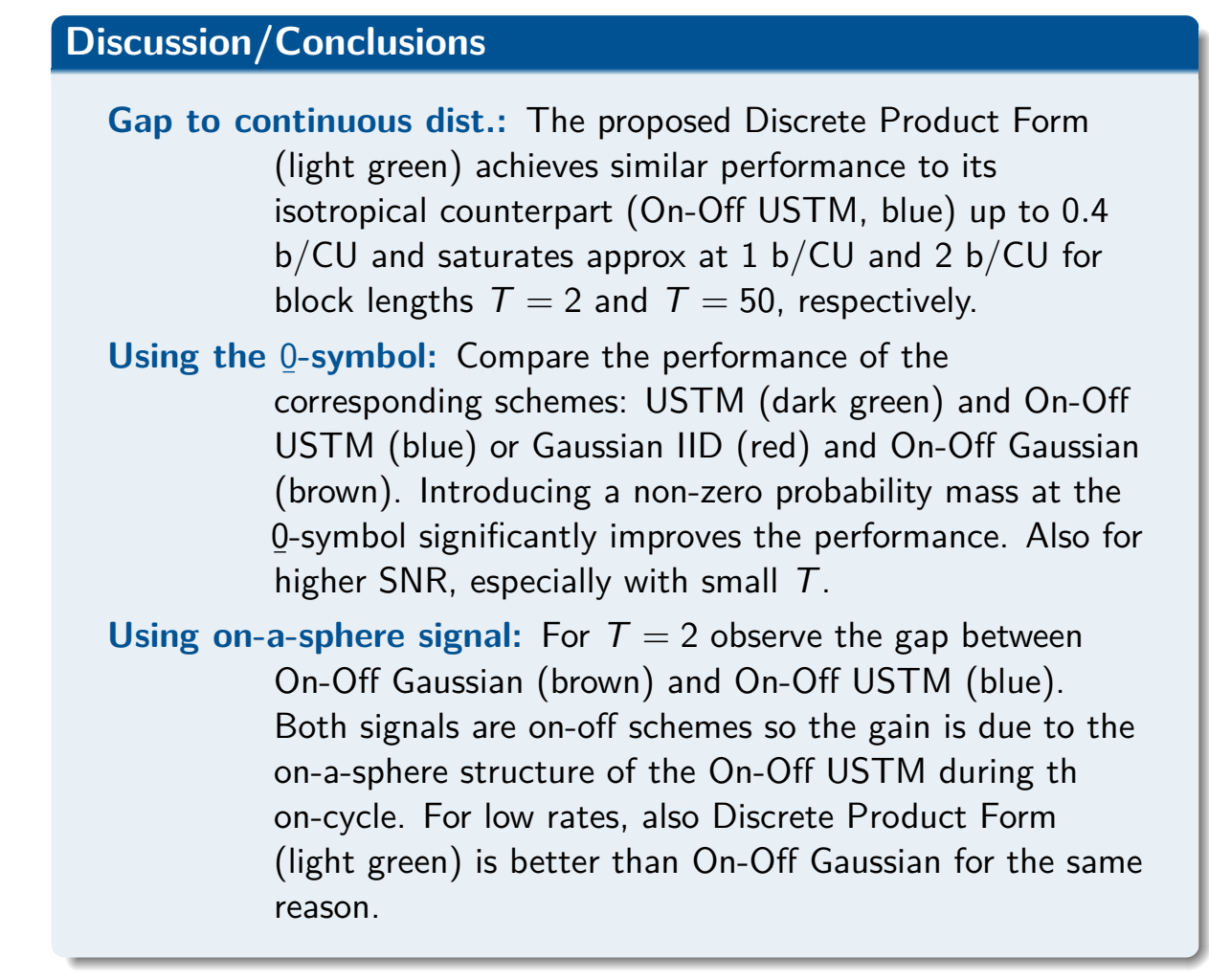

\section{References}

1] F. Rusek, A. Lozano, and N. Jindal, "Mutual information of IID complex
Gaussian signals on llock Rayleigh-faded channels," IEEE Trans. Inf. Theory vol. 58, no. 1, pp. 331-340, Jan. 2012

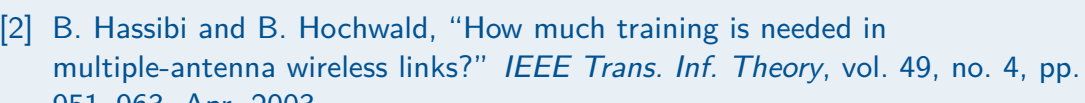

B. Hochwald and T. Marzetta, "Unitary space-time modulation for
multiple-antenna communications in Rayleigh flat fading," IEEE Trans. Inf

[4] M. Pikus, G. Kramer, and G. Bocherer, "Discrete signaling for non-coherent,
single-artenana, aryleign block-fading channess," IIEEE Communications
Letters, vol. PP, no. 99, pp. 1-1, 2016. 